\title{
Diastolic dysfunction in rheumatoid arthritis: a usual travel-mate?
}

\author{
Ilaria Cavazzana ${ }^{1}$, Enrico Vizzardi², Franco Franceschini ${ }^{1,3}$ \\ ${ }^{1}$ Rheumatology Unit, ASST Spedali Civili, Brescia; ${ }^{2}$ Section of cardiovascular disease, ASST Spedali Civili, \\ Department of Medical and Surgical Specialties, Radiological Sciences and Public Health, University of Brescia; \\ ${ }^{3}$ Experimental and Clinical Science Department, University of Brescia, Italy
}

\begin{abstract}
A high rate of left ventricular diastolic dysfunction (LVDD) has been described in rheumatoid arthritis (RA), compared with general population. A prospective study demonstrated that LVDD occurred in $24 \%$ in one year of follow-up in RA patients without cardiac disease. Older age, higher systolic arterial pressure and lower E/A ratio are considered predictive factors. In addition, in RA, LVDD is known to be the only risk factor for the development of cardiovascular (CV) events, also in absence of classical CV risk factors. Some occasional reports suggest that early and aggressive treatment of RA could influence the evolution of LVDD and, accordingly, modify the rate of $\mathrm{CV}$ events. Therefore, a correct assessment of diastolic function should be considered of pivotal importance in the routine follow-up of RA patients.
\end{abstract}

Rheumatoid arthritis (RA) is known to impact on diastolic heart function. A high rate of left ventricular diastolic dysfunction (LVDD) has been described in RA, compared with general population, without consistent association with disease activity or duration [1].

LVDD encompasses common structural ventricular abnormalities, such as decreased distensibility, impaired relaxation, abnor-

\footnotetext{
Correspondence: Ilaria Cavazzana, UO Reumatologia e Immunologia Clinica, ASST Spedali Civili, piazzale Spedali Civili 1, 25124 Brescia, Italy. Tel. +39.030.3995488-1 - Fax: +39.030.3995085. Email: ilariacava@virgilio.it
}

Key words: Diastolic dysfunction; rheumatoid arthritis; cardiovascular events.

Authors' contribution: FF, manuscript concept and design, first draft revision and corrections; IC,EV, manuscript drafting.

Conflict of interest: the authors declare no conflict of interest.

Funding: AbbVie provided funding for medical writing (protocol number: 0068880)

Received for publication: 23 July 2019.

Accepted for publication: 26 July 2019.

${ }^{\circ}$ Copyright: the Author(s), 2019

Licensee PAGEPress, Italy

Monaldi Archives for Chest Disease 2019; 89:1137

doi: 10.4081/monaldi.2019.1137

This article is distributed under the terms of the Creative Commons Attribution Noncommercial License (by-nc 4.0) which permits any noncommercial use, distribution, and reproduction in any medium, provided the original author(s) and source are credited. mal diastolic filling, usually with normal ejection fraction [2]. The pathogenesis of LVDD in RA is not actually yet elucidated: some author hypothesized a chronic myocarditis and hypoperfusion leading to a lower LV mass, as demonstrated by magnetic resonance studies [3]. Amyloidosis has been considered as another potential trigger of abnormal ventricular diastolic function [4]. The preservation of ejection fraction and the lower rate of known cardiovascular $(\mathrm{CV})$ risk factors in RA patients with LVDD compared to general population [1] suggest that the inflammatory burden could play a role inducing myocardial stiffening and progressively leading to moderate/severe $\operatorname{LVDD}[5,6]$.

In volume 89, issue 3 of the Monaldi Archives for Chest Disease, dal Piaz et al. published a prospective study analysing the occurrence of LVDD in asymptomatic patients with RA followed for 1 year. After 1 year, LVDD was detected in $24 \%$ of RA patients without overt cardiac disease: a lower E/A ratio of transmitral flow, older age and higher systolic blood pressure were independently associated with new-onset LVDD. A simple clinical model is proposed to predict the development of LVDD, including 57 years of age; $131 \mathrm{mmHg}$ of systolic arterial pressure and 0.96 of E/A ratio of transmitral flow. The accuracy of the proposed model showed moderate sensitivity and specificity but high negative predictive value. In addition to the prediction model, the original data stands on the high annual rate of new onset of LVDD in patients asymptomatic for CV disease. Authors reported two main limitations, represented by the inclusion of patients with known $\mathrm{CV}$ risk factors and the impact of pharmacologic treatments for RA (e.g., Plaquenil, steroids, NSAIDs, Methotrexate, immunomodulant agents) on LVDD development.

LVDD alone is known to be responsible of increased adverse $\mathrm{CV}$ events in the general population $[7,8]$ and in patients with cardiopathy $[9,10]$.

In order to evaluate the potential effect of LVDD on the new onset of CV events in RA patients without CV risk factors, we previously compared 19 cases with LVDD and 9 patients without LVDD. After 7 years of follow-up, CV events occurred only in the first group (LVDD+ CV- patients) ( 7 cases; $\mathrm{p}=0.043$ ), represented by congestive heart failure, coronary bypass graft surgery, transient ischemic attack and new onset arterial hypertension [11]. In this little monocentric paper, LVDD demonstrates to be the only risk factor for the development of $\mathrm{CV}$ events, also in absence of classical CV risk factors. The natural history of LVDD is not completely clarified: in general population LVDD remained stable in $50 \%$ of cases and deteriorated in about one-third in 3-6 years of follow-up [12]. In another case-series the 2-year probability of heart failure is $1.9 \%$ [13].

Little is known on the natural history of LVDD in RA patients and very few data are available regarding the potential effects of different disease modifying anti-rheumatic drugs (DMARDs) or biological drugs on the evolution of LVDD. 
A significant improvement in diastolic function has been demonstrated by cardiac magnetic resonance after 1 year of DMARDs treatment in new-onset RA patients, suggesting that early treatment of RA could decrease systemic inflammation [14]. Few data are available on the effects of different immunosuppressive drugs on myocardial function. A comparison between methotrexate and tocilizumab (e.g., a biological agent inhibiting IL-6 function) demonstrated a normalization of myocardial dysfunction, assessed by cardiac magnetic resonance, in a little group of RA: in particular, the mean peak radial strain was lower in patients treated by Methotrexate, while patients treated by tocilizumab showed higher values, comparable with normal controls [15]. More recently other authors reported a reversal in baseline subclinical CV dysfunction, namely myocardial inflammation, functional strain parameters, and aortic stiffness, after 6 months of treatment with anti-TNF agents [16].

These scanty data suggest that early and aggressive treatment of RA could influence the evolution of LVDD and, accordingly, modify the rate of CV events. In addition, the rapid onset and the high rate of new LVDD in RA, as depicted in the paper of dal Piaz et al., outline how the assessment of diastolic function should be considered of pivotal importance in the routine follow-up of RA patients.

\section{References}

1. Aslam F, Bandeali SJ, Khan NA, Alam M. Diastolic dysfunction in rheumatoid arthritis: a meta-analysis and systematic review. Arthritis Care Res 2013; 65:534-43.

2. Fukuta H, Littel WC. Diagnosis of diastolic heart failure. Curr Cardiol Rep 2007;9:224-8.

3. Giles JT, Malayeri AA, Fernandes V, et al. Left ventricular structure and function in patients with rheumatoid arthritis, as assessed by cardiac magnetic resonance imaging. Arthritis Rheum 2010;62:940-51.

4. Koivuniemi R, Paimela L, Suomalainen R, et al. Amyloidosis is frequently undetected in patients with rheumatoid arthritis. Amyloid 2008;15:262-8.

5. Davis JM 3rd, Knutson KL, Strausbauch MA, et al. A signature of aberrant immune responsiveness identifies myocardial dysfunction in rheumatoid arthritis. Arthritis Rheum 2011;63:1497-506.
6. Giles JT, Fernandes V, Lima JA, et al. Myocardial dysfunction in rheumatoid arthritis: epidemiology and pathogenesis. Arthrits Res Ther 2005;7:195-207.

7. Mogelvang R, Sogaard P, Pedersen SA, et al. Cardiac dysfunction assessed by echocardiographic tissue Doppler imaging is an independent predictor of mortality in the general population. Circulation 2009; 119:2679-85.

8. Mogelvang R, Biering-Sorensen T, Jensen JS. Tissue Doppler echocardiography predicts acute myocardial infarction, heart failure, and cardiovascular death in the general population. Eur Heart J Cardiovasc Imaging 2015;16:1331-7.

9. Mishra RK, Devereux RB, Cohen BE, et al. Prediction of heart failure and adverse cardiovascular events in outpatients with coronary artery disease using mitral E/A ratio in conjunction with e-wave deceleration time: the heart and soul study. J Am Soc Echocardiogr 2011;24:1134-40.

10. Johansen ND, Biering-Sorensen T, Jensen JS, Mogelvang R. Diastolic dysfunction revisited: A new, feasible, and unambiguous echocardiographic classification predicts major cardiovascular events. Am Heart J 2017;118:136-46.

11. Vizzardi E, Bazzani C, Cavazzana I, et al. Prognostic value of diastolic dysfunction in asymptomatic Rheumatoid Arthritis patients without cardiovascular risk factors. Clin Exp Rheumatol 2016;34:352.

12. Achong N, Wahi S, Marwick TH. Evolution and outcome of diastolic dysfunction. Heart 2009;95:813-8.

13. Correa de Sa DD, Hodge DO, Slusser JP, et al. Progression and preclinical diastolic dysfunction to the development of symptoms. Heart 2010;96:528-32.

14. Lehmonen L, Vuorinen AM, Koivuniemi R, et al. One-year follow-up study detects myocardial changes with cardiovascular magnetic resonance tagging in active Rheumatoid Arthritis. Acad Radiol 2018;25:476-85.

15. Kobayashi Y, Kobayashi H, Hirano M, Giles JT. Left ventricular regional dysfunction using cardiac magnetic resonance imaging in rheumatoid arthritis patients without cardiac symptoms: comparison between methotrexate and biologics treatment groups. J Rheumatol 2014;41:1560-2.

16. Ntusi NAB, Francis JM, Sever E, et al. Anti-TNF modulation reduces myocardial inflammation and improves cardiovascular function in systemic rheumatic diseases. Int $\mathrm{J}$ Cardiol 2018;270:253-9. 